\title{
Use of the beta-phase of Poly(9,9-dioctylfluorene) as a Probe into the Interfacial Interplay for the Mixed Bilayer Films \\ Formed by Sequential Spin-coating
}

Rui Zhu ${ }^{\dagger}, *$, Jian-Ming Lin ${ }^{\dagger}$, Wei-Zhi Wang ${ }^{\dagger}$, Chao Zheng ${ }^{\dagger}$, Wei Wei ${ }^{\dagger}$, Wei Huang ${ }^{\dagger+, * *}$, Yun-Hua Xu ${ }^{\S}$, Jun-Biao Peng ${ }^{\S}$, and Yong $\mathrm{Cao}^{\S}$

${ }^{\dagger}$ Institute of Advanced Materials (IAM), Fudan University, 220 Handan Road, Shanghai 200433, China

* Institute of Advanced Materials (IAM), Nanjing University of Posts and Telecommunications, 66 XinMoFan Road, Nanjing 210003, China

$\S$ Institute of Polymer Optoelectronic Materials and Devices, Key Laboratory of Specially Functional Materials and Advanced Manufacturing Technology, South China University of Technology, Guangzhou 510640, China

*Corresponding authors'E-mail: (R.Z.) iamzhurui@gmail.com ; (W.H.) wei-huang@njupt.edu.cn 


\section{The method to determine the thickness of PVK films}

Thickness of PVK layer was measured by Tencor Alphastep surface profilometer accompanied with the measurement of film absorption intensities. PVK films on glass substrate with different thickness were obtained by varying the concentration of PVK solutions. The thickness was firstly measured by Tencor Alphastep surface profilometer. UV-vis absorption spectra were then taken for these films, as displayed in Figure S1. The absorption intensity at $345 \mathrm{~nm}$ was recorded in Table S1 for films with different thickness. Absorption intensity at $345 \mathrm{~nm}$ was then plotted versus to the corresponding film thickness measured by Tencor Alphastep surface profilometer. A linear fit through zero point was made for these data to obtain the correlation between thickness and absorption at $345 \mathrm{~nm}$, as displayed in Figure S2. Equation for this linear fit result is:

Absorption intensity (a.u.) $=0.0023 \times$ Thickness $(\mathrm{nm})$

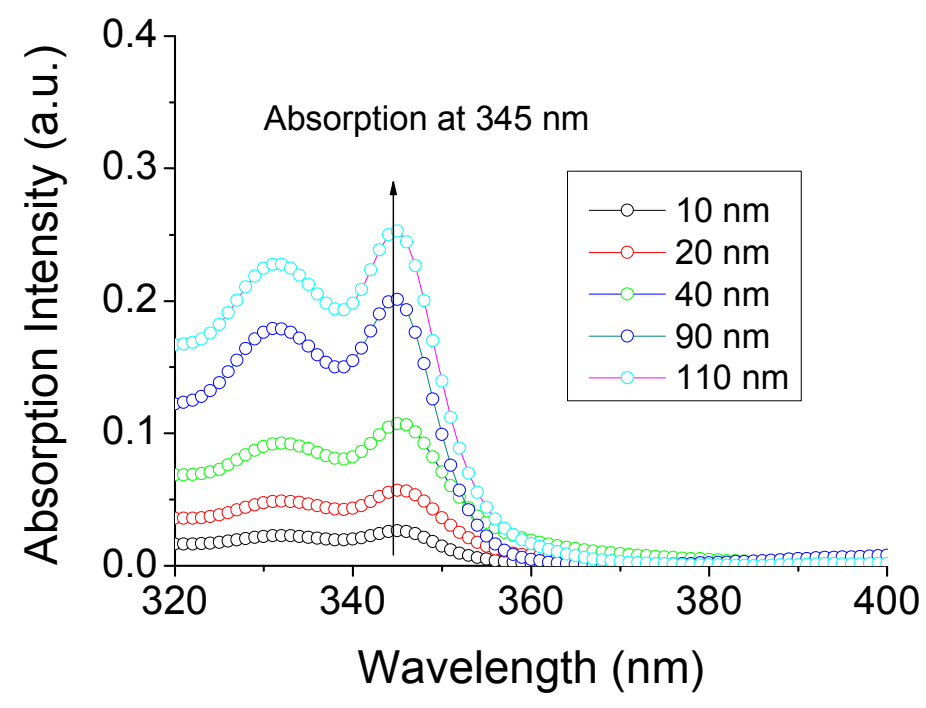

Figure S1. UV-vis absorption of PVK layers with varied thickness. 
Table S1. Film thickness and the corresponding absorption intensity

\begin{tabular}{cc}
\hline $\begin{array}{c}\text { Film Thickness } \\
(\mathrm{nm})\end{array}$ & $\begin{array}{c}\text { Absorption intensity at } 345 \mathrm{~nm} \\
\text { (a.u.) }\end{array}$ \\
\hline 10 & 0.026 \\
20 & 0.057 \\
40 & 0.11 \\
90 & 0.20 \\
110 & 0.25 \\
\hline
\end{tabular}

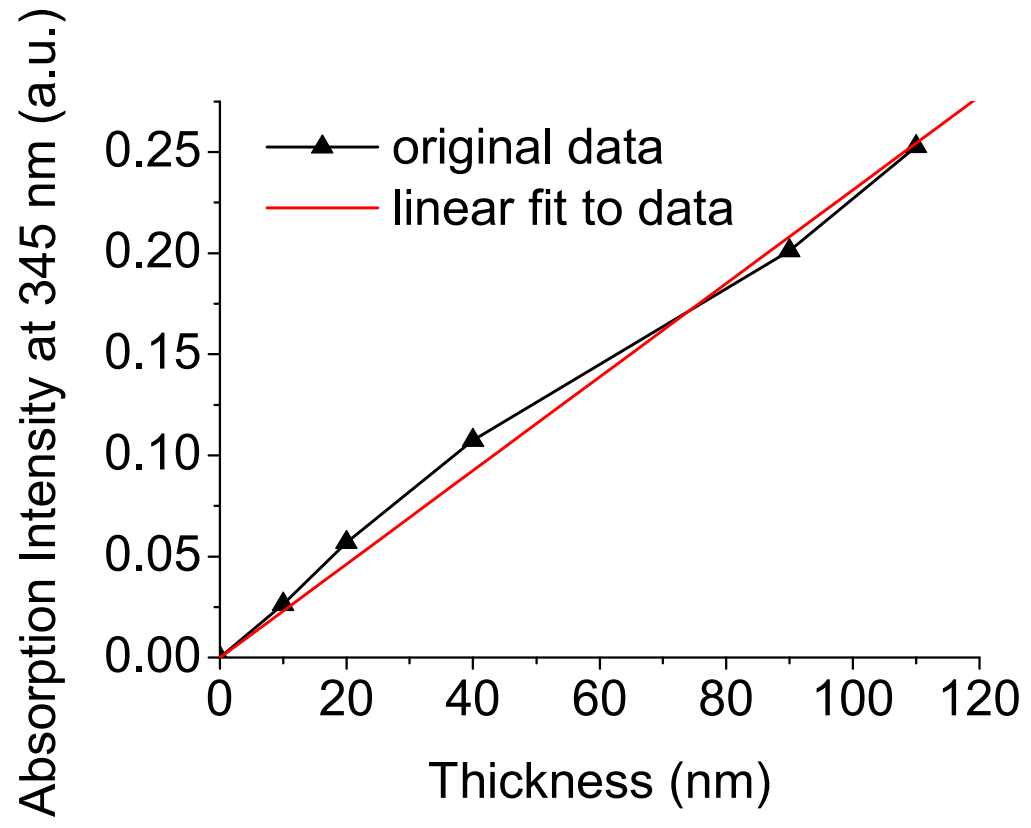

Figure S2. Correlation between the absorption intensity at $345 \mathrm{~nm}$ and the thickness for PVK films. Linear fit through the zero point was made for these data. 


\section{Residual film thickness after washing PVK films by pure toluene}

Pure toluene was dripped onto the deposited PVK films with different initial thickness. Spinning process was then preceded at a rate of 1300 rpm. This process can be called washing. The UV-vis absorption spectra of the washed films were shown in Figure S3. The absorption intensity at $345 \mathrm{~nm}$ for these washed films was recorded in Table S2. Based on these data, thickness of residual PVK layers can be estimated according to Eq. (1). It is obvious that, when the initial thickness of PVK layer was 100 or $200 \AA$, the residual PVK was hardly observed. However, for the initial thickness of about or above $400 \AA$, residual PVK layers could be observed clearly. This result implies that, most of the deposited PVK layer will be washed off during the second spin-coating process.

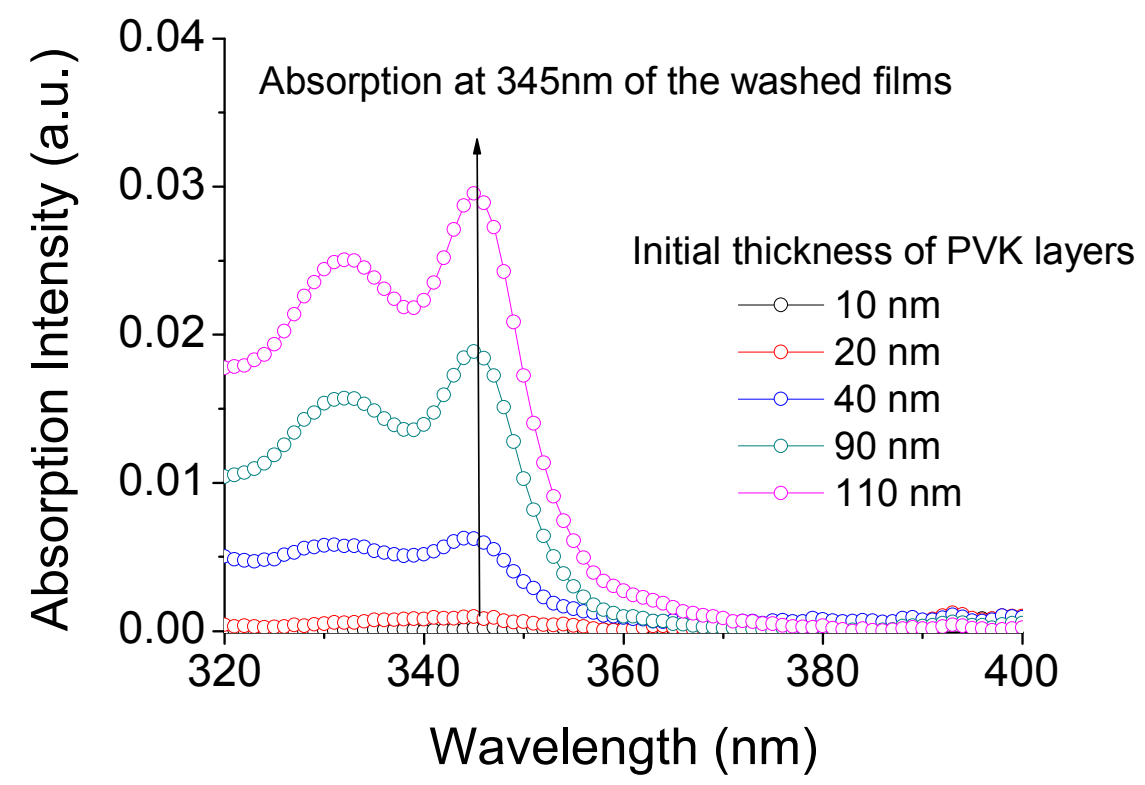

Figure S3. UV-vis absorption intensity of the PVK films after washing with pure toluene. 
Table S2. Thickness estimation for the PVK films after washing. Initial Thickness Absorption intensity Approximate

(§̊) of the PVK films after thickness of the PVK washing (a.u.) films after washing

\begin{tabular}{ccc} 
& & $(\AA)$ \\
\hline 100 & 0.00015 & $\sim 1$ \\
200 & 0.00095 & $\sim 4$ \\
400 & 0.0062 & $\sim 30$ \\
900 & 0.019 & $\sim 80$ \\
1100 & 0.030 & $\sim 130$ \\
\hline
\end{tabular}

